OPEN ACCESS

Citation: Ian M. Turner (2020) A synopsis of the native Combretaceae in the Malay Peninsula. Webbia. Journal of Plant Taxonomy and Geography 75(2): 263-280. doi: 10.36253/jopt-8891

Received: May 14, 2020

Accepted: July 6, 2020

Published: November 18, 2020

Copyright: (c) 2020 lan M. Turner. This is an open access, peer-reviewed article published by Firenze University Press (http://www.fupress.com/webbia) and distributed under the terms of the Creative Commons Attribution License, which permits unrestricted use, distribution, and reproduction in any medium, provided the original author and source are credited.

Data Availability Statement: All relevant data are within the paper and its Supporting Information files.

Competing Interests: The Author(s) declare(s) no conflict of interest.

Editor: Peter C. Boyce

\section{A synopsis of the native Combretaceae in the Malay Peninsula}

\author{
IAN M. TURNER \\ Singapore Botanical Liaison Officer, Royal Botanic Gardens, Kew, Richmond, Surrey \\ TW9 3AE, UK; Herbarium, Singapore Botanic Gardens, National Parks Board, 1 Cluny \\ Road, Singapore 259569 \\ E-mail: i.turner@kew.org
}

\begin{abstract}
A synopsis of the native species of Combretaceae in the Malay Peninsula (Peninsular Thailand, Peninsular Malaysia and Singapore) is presented. A total of 29 species in four genera (Combretum, Getonia, Lumnitzera and Terminalia) are recognised. Keys to genera and species are included with synonymy and typification. In total 63 lectotypifications, three second-step lectotypifications and 11 neotypifications are proposed in the paper.
\end{abstract}

Keywords: Calycopteris, Combretum, Getonia, Lumnitzera, Quisqualis, Terminalia.

\section{INTRODUCTION}

The Combretaceae include about 500 species of predominantly trees and scandent shrubs found across most of the equatorial belt (Stace 2007). The family is well defined and its circumscription and higher-level classification is uncontentious. It is numerically dominated by two large genera Combretum and Terminalia which together make up about $97 \%$ of the extant species (Stace 2010). Combretaceae species are found in tropical rain forests, dry forests, savanna woodlands, savannas, littoral and mangrove vegetation. They are less common in montane forests.

In South-East Asia the family is a member of the native flora and its species are encountered in the lowlands. For the Malay Peninsula, the taxonomy of the family was covered by Ridley (1922) and Exell (1954). Since then there have been revisions of Combretum and Terminalia in Thailand (Nanakorn 1985, 1986), and of the arborescent taxa in Peninsular Malaysia (Kochummen 1972).

Recent activity in Combretaceae systematics has largely concerned generic circumscription and the general trend has been for the subsumation of small genera into the big two (Combretum and Terminalia). This can be epitomised by a comparison between the system proposed by Exell and Stace (1966) that recognised 20 genera and a recent summary (Byng 2014) where this number had dropped to 10. These changes are less evident in South-East 
Asia where the only major change is the inclusion of Quisqualis in Combretum (Maurin et al. 2020).

The purpose of this study is to provide a taxonomic overview of the family as represented in the native flora of the Malay Peninsula including keys to genera and species, a nomenclatural synopsis and details of typification. The Malay Peninsula is taken here to include Peninsular Thailand, Peninsular Malaysia and Singapore. The research was conducted with reference to the collections in herbarium of the Royal Botanic Gardens Kew $(\mathrm{K})$, some specimens on loan from SING, visits to $\mathrm{BM}$ and access to various internet databases (e.g. JSTOR Global Plants) and virtual herbaria (e.g. Berlin, Geneva, Paris).

\section{KEY TO GENERA OF COMBRETACEAE IN THE MALAY PENINSULA}

1. Mangrove trees with reddish twigs, fleshy leaves with a gland on the abaxial surface just below the apical notch; flowers and fruits bearing a pair of bracteoles adnate to the hypanthium. Lumnitzera

Trees and scandent shrubs of seashores and inland forests, not true mangroves, twigs green or brown, not notably reddish, leaves chartaceous to coriaceous, not fleshy, lacking an apical gland; flowers and fruits without adnate bracteoles. . .2

2. Scandent shubs (except C. quadrangulare); flowers petalous. Combretum

Erect trees and treelets; flowers apetalous .3

3. Sepals accrescent in fruit. Getonia Sepals not persisting in fruit. Terminalia

1. Combretum Loefl., Iter Hispan. 308. 1758, nom. cons.

Type: Combretum fruticosum (Loefl.) Stuntz

(三) Aetia Adans., Fam. Pl. 2: 84, 513. 1763, nom. illegit. superfl.

(=) Grislea L., Sp. Pl. 1: 348. 1753, nom. rejic. Type: Grislea secunda L.

(=) Quisqualis L., Sp. Pl. (ed. 2) 1: 556. 1762. - Udani Adans., Fam. Pl. 2: (22). 1763, nom. illegit., superfl.. - Combretum sect. Quisqualis (L.) Stace, Fl. Neotrop. Monogr. 107: 159. 2010.

Type: Quisqualis indica L.
(=) Kleinia Crantz, Inst. Rei Herb. 2: 488. 1766, nom. illegit., non Kleinia Mill. (1754), nec Kleinia Jacq. (1760).

Type: Kleinia quadricolor Crantz.

(=) Cacoucia Aubl., Hist. Pl. Guiane 1: 450, pl. 179. 1775. - Hambergera Scop., Introd. (1777) 106. 1777, nom. illegit. superfl. - Schousboea Willd., Sp. Pl. 2: 578. 1799, nom. illegit. superfl. - Combretum subgenus Cacoucia (Aubl.) Exell \& Stace, Bol. Soc. Brot., Sér. 2, 40: 10. 1966. Type: Cacoucia coccinea Aubl.

(=) Cristaria Sonn., Voy. Ind. Orient. 2: 247. 1782, nom. rejic. - Poivrea Comm. ex DC., Prodr. 3: 17. 1828, nom. illegit. superfl. - Combretum sect. Poivrea G. Don, Gen. Hist. 2: 665. 1832.

Type: Cristaria coccinea Sonn.

(=) Sphalanthus Jack, Malayan Misc. 2(7): 55. 1822. Quisqualis sect. Sphalanthus (Jack) Exell, J. Bot. 69: 121. 1931.

Type: Sphalanthus confertus Jack.

(=) Gonocarpus Ham., Prodr. Pl. Ind. Occid. 39. 1825, nom. illegit., non Gonocarpus Thunb. (1783).

Type: Gonocarpus jacquinii Ham., nom. illegit. superfl.

(=) Forsgardia Vell., Fl. Flumin. 4: 152, t. 13. 1829.

Type: Forsgardia laevis Vell.

(=) Chrysostachys Pohl, Pl. Bras. Icon. Descr. 2: 66, t. 143. 1830.

Type: Chrysostachys ovatifolia Pohl.

(=) Sheadendron G.Bertol., Mem. Reale Accad. Sci. Ist. Bologna 2: 574. 1850.

Type: Sheadendron butyrosum G.Bertol.

(=) Calopyxis Tul., Ann. Sci. Nat., Bot., sér. 4, 6: 86. 1856. - Combretum sect. Calopyxis (Tul.) Jongkind, Bull. Mus. Natl. Hist. Nat., B, Adansonia Sér. 4, 17(3-4): 193. 1995.

Type: Calopyxis sphaeroides Tul. (lectotype selected by Stace (2010: 60).

(=) Embryogonia Blume, Mus. Bot. 2: 122. 1856.

Type: Embryogonia acuminata (Roxb.) Blume (lectotype selected by Stace (2010: 60).

(=) Bureava Baill., Adansonia 1: 71. 1860. 71, nom. rejic. Type: Bureava crotonoides Baill.

(=) Thiloa Eichler, Flora 49: 149. 1866. - Combretum sect. Thiloa (Eichler) Stace, Fl. Ecuador 81: 13. 2007. 
Type: Thiloa glaucocarpa (Mart.) Eichler (lectotype selected by Stace, Fl. Ecuador 81: 11. 2007).

(=) Campylogyne Welw. ex Hemsl., Hooker's Icon. Pl. 26: t. 2550. 1889. - Quisqualis sect. Campylogyne (Welw. ex Hemsl.) Exell, J. Bot. 69: 120. 1931.

Type: Campylogyne exannulata (O.Hoffm.) Hemsl.

(=) Campylochiton Welw. ex Hiern, Cat. Afr. Pl. 2: 353. 1901.

Type: Campylochiton platypterus (Welw.) Hiern.

(=) Meiostemon Exell \& Stace, Bol. Soc. Brot., sér. 2, 40: 18. 1966.

Type: Meiostemon tetrandrus (Exell) Exell \& Stace

Notes

The lectotypifications of Calopyxis and Thiloa were first proposed by Exell (1931) but the choices were clearly mechanical, so the later designations by Stace (2007, 2010) are accepted here.

\section{Key to Combretum taxa}

1. Floral bracts persisting to anthesis and beyond; upper hypanthium tubular; petals becoming pink or red

Floral bracts generally dropping before anthesis; upper hypanthium salveriform to infundibuliform; petals white or green

2. Upper hypanthium 5-8 cm long 1.5 C. indicum

Upper hypanthium 1-2 cm long .3

3. Sepals recurved at anthesis, with filiform tips $1-2 \mathrm{~mm}$ long 1.4 C. densiflorum

Sepals not recurved at anthesis, filiform tips to $1 \mathrm{~mm}$ long.

4. Leaves with tertiary venation visible adaxially; floral bracts to $2 \mathrm{~mm}$ long with 1 central longitudinal nerve. 1.6 C. langkawiense

Leaves with tertiary venation obscure adaxially; floral bracts $1 \mathrm{~cm}$ long or more with at least 5 longitudinal nerves. .1.2 C. caudatum

5. Indumentum including glandular hairs but not scales; flowers 5-merous; fruits with 5 wings or 5 longitudinal ridges (except $C$. nigrescens which has 4 -merous flowers and 4-winged fruits)

Indumentum including scales but not glandular hairs; flowers 4-merous; fruits with 4 wings or 4 longitudinal ridges
6. Flowers 5-merous; fruits with 5 wings or 5 longitudinal ridges

Flowers 4-merous; fruits with 4 wings or 4 longitudinal ridges 1.8 C. nigrescens

7. Leaves opposite with hairy domatia in axils of lateral nerves below; fruits with 5 wings 1.12 C. roxburghii

Leaves often ternate without domatia; fruits with 5 longitudinal ridges 1.15 C. trifoliatum

8. Trees, young twigs sharply 4-angled.1.11 C. quadrangulare Climbers or scandent shrubs, young twigs more or less terete 9

9. Lower lamina with a dense covering of predominantly whitish scales, tertiary venation obscure 10

Lower lamina with scales absent or with sparse covering of predominantly reddish scales, tertiary venation visible... 11

10. Inflorescences subcapitate. 1.10a C. punctatum var. punctatum

Inflorescences elongate

1.10b C. punctatum var. squamosum

11. Petals inconspicuous, to $1.5 \mathrm{~mm}$ long; fruits with 4 longitudinal blunt-topped ridges ..... 1.1 C. acuminatum

Petals conspicuous at anthesis, more than $1.5 \mathrm{~mm}$ long; fruits with 4 distinct wings or if with longitudinal ridges then ridges sharp-edged (C. tetralophum)

12. Sepals $1.5 \mathrm{~mm}$ or longer and petals exceeding length of sepals; fruit with 4 longitudinal sharp-edged ridges 1.14 C. tetralophum

Sepals to $1 \mathrm{~mm}$ long, or if longer then petals shorter than sepals; fruits with 4 papery wings. 13

13. Inflorescences capitate (also detectable as knobbly ends to infructescences 3-4 $\mathrm{mm}$ long) 1.13 C. sundaicum

Inflorescences elongate 14

14. Flowers and inflorescence rhachis densely pale erect hairy; fruits sparsely hairy, scales absent. 1.7 C. latifolium

Flowers and inflorescence rhachis densely scaly; fruits glabrous but with scales present

15. Twigs drying reddish brown; leaves with red-brown scales conspicuous on lower lamina; flowers generally more than $4 \mathrm{~mm}$ long; fruits generally more than $3 \mathrm{~cm}$ across... 1.3 C. chinense

Twigs drying whitish; leaves without conspicuous redbrown scales on lower lamina; flowers generally less than 4 $\mathrm{mm}$ long; fruits to $2.5 \mathrm{~cm}$ across. ..1.9 C. porterianum 
1.1 Combretum acuminatum Roxb., Fl. Ind. 2: 228. 1832

Type: [unpublished illustration] Icones Roxburghianae 2225 (K, lectotype selected by du Puy and Telford (1993: 245).

(三) Embryogonia acuminata (Roxb.) Blume, Mus. Bot. 2: 123. 1856.

(=) Combretum costatum Roxb., Fl. Ind. 2: 227. 1832.

Type: [unpublished illustration] Icones Roxburghianae 2478 (2476 on drawing) (lectotype CAL, selected by Gangopadhyay and Chakrabarty (1997: 299).

(=) Combretum neurophyllum Miq., Fl. Ned. Ind. 1(1): 608. 1856.

Type: Java, Tjitjiringin, J. Hasskarl s.n. (L barcode L0844403, lectotype designated here).

(=) Combretum sarcopterum Thwaites, Enum. Pl. Zeyl. 415. 1864.

Type: Sri Lanka, Reigam Corle, Thwaites CP 3715 (CAL, lectotype selected by Gangopadhyay and Chakrabarty (1997: 299).

(=) Combretum stenopetalum Van Heurck \& Müll.Arg., Observ. Bot. Descript. Pl. Nov. Herb. Van Heurckiani 2: 225. 1871.

Type: W. Griffith (syntypes BR, G).

(=) Combretum vanheurckii Müll.Arg., Observ. Bot. Descript. Pl. Nov. Herb. Van Heurckiani 2: 227. 1871.

Type: W. Griffith 725 (G barcode G00177909, lectotype selected by Gangopadhyay and Chakrabarty (1997: 299); isolectotype BR).

(=) Terminalia borneensis Ridl., Bull. Misc. Inform. Kew 1938: 283. 1938, nom. illegit., non T. borneensis Slooten (1919). Type: Borneo, Bangarmassing, J. Motley 380 (K barcode K000786585, lectotype designated here).

Notes

Nanakorn (1986: 196) referred to a specimen in G-DC from the distribution of the East India Company Herbarium under number $4014 \mathrm{D}$ as type of Combretum acuminatum Roxb. Some error crept in here - Nanakorn's proposed type is a specimen of Anogeissus acuminata (Roxb. ex DC.) Wall. ex Guillem. \& Perr. rather than Combretum, and is certainly not original material for Roxburgh's name in Combretum. Therefore, the later typification by du Puy and Telford to a Roxburgh drawing is accepted here.
1.2 Combretum caudatum (Craib) O.Maurin \& Boatw., Phytotaxa 451: 232. 2020

Bas.: Quisqualis caudata Craib, Bull. Misc. Inform. Kew 1930: 164. 1930.

Type: Thailand, Ranawng, Kao Pawta, Chongdong, 21 January 1929, A.F.G. Kerr 16797 (K barcode K000786136, lectotype selected by Maurin et al. (2020: 232); isolectotypes ABD, BK barcode BK257801, BM barcode BM000947035).

\section{Notes}

Maurin et al. (2010) included this species in their analysis that showed it to be sister to the other 'Quisqualis' species sampled and to be part of a subclade of Combretum subgenus Cacoucia.

1.3 Combretum chinense Roxb. ex G.Don, Trans. Linn. Soc. 15: 417, 432-433. 1827

Type: Calcutta Botanical Garden, W. Roxburgh s.n. (G barcode G00177910, lectotype selected by Nanakorn (1986: 174).

(=) Combretum chinense Roxb., Fl. Ind. 2: 230. 1832, as 'chinensis', nom. illegit., non C. chinense Roxb. ex G.Don (1827).

Type: India, Calcutta Botanical Garden, W. Roxburgh s.n. (G barcode G00177910, lectotype selected by Nanakorn (1986: 174).

(=) Combretum chinense Roxb. var. pubescens King, J. Asiat. Soc. Bengal, Pt. 2, Nat. Hist. 66: 339. 1897.

Type: Peninsular Malaysia, Perak, Sungai Ryah, October 1880, Dr King's Collector 889 (K barcode K001129737, lectotype designated here).

(=) Combretum annulatum Craib, Bull. Misc. Inform. Kew 1929: 113. 1929.

Type: Thailand, Chumpawn, Ta Ngaw, 17 January 1917, A.F.G. Kerr 11488 (K barcode K000786590, lectotype selected by Gangopadhyay and Chakrabarty 1997: 314; isolectotypes ABD, BK barcode BK257787, BM barcode BM000947049, TCD barcode TCD0015621).

(=) Combretum annulatum Craub var. orbiculare Craib, Fl. Siam. Enum. 1(4): 614. 1931.

Type: Thailand, Puket, Ranawng, Kampuam, 29 January 1929, A.F.G. Kerr 16881 (K (2 sheets) barcodes K000786589, K001129727, lectotype designated here; isolectotypes ABD, BM barcode BM000947050). 
Notes

There has been considerable confusion about the name Combretum chinense. It was first published by William Roxburgh in his Hortus Bengalensis (Roxburgh 1814) but was a nomen nudum in the absence of any description or reference to a published description. As with many Roxburgh names, it was first validated by another author before it appeared with a description in one of the publications of Roxburgh's works after his death. George Don described Combretum chinense Roxb. in 1827 (Don 1827), before Roxburgh's own description was finally published in 1832 (Roxburgh 1832). Don referred to a Roxburgh specimen in the herbarium of A.B. Lambert. There is a relevant Roxburgh specimen now in $G$ that appears to be annotated by Lambert. Exell (1954) argued that this was not Don's type as it did not match all the details in Don's description. He therefore treated C. chinense Roxb. ex G.Don as a confused name and referred the Malesian material to Combretum yunnanese Exell. Nanakorn (1985) disagreed, and effectively lectotypified both the Don and Roxburgh names to the G specimen. I find that the Malay Peninsula material is a reasonable match to the Roxburgh lectotype (image available online) and therefore employ $C$. chinense Roxb. ex G.Don for the specimens.

1.4 Combretum densiflorum (Wall. ex Planch.) I.M.Turner, Phytotaxa 451: 232. 2020

Bas.: Quisqualis densiflora Wall. ex Planch., Fl. Serres 6: 348. 1851.

Type: Peninsular Malaysia, Penang, G. Porter s.n. [EIC 4011] (K-W barcode K001117910, lectotype selected by Maurin et al., 2020: 232; isolectotypes BR barcode BR0000005639281, GZU barcode GZU000273166, K barcode K000786138, M barcode M0146742, P (×3) barcodes P01901448, P01901447, P01901446, PH barcode PH00023582

(=) Sphalanthus confertus Jack, Malayan Misc. 2(7): 55-56. 1822. - Quisqualis conferta (Jack) Exell, J. Bot. 69: 122. 1931.

Type: W. Jack s.n. (G barcode G00446750, lectotype selected by Exell (1931: 122).

Notes

The oldest name for this species is Sphalanthus confertus Jack, but Combretum confertum (Benth.) M.A.Lawson, the accepted name of an African species, blocks the transfer of Jack's name to Combretum.
1.5 Combretum indicum (L.) DeFilipps, Useful Pl. Dominica 277. 1998

Bas. Quisqualis indica L., Sp. Pl., ed. 2. 1: 556. 1762.

Type: [published illustration] "Quis qualis" in Rumphius, Herb. Amboin. 5: t. 38.1747 (lectotype selected by Merrill (1917: 390).

(=) Kleinia quadricolor Crantz, Inst. Rei Herb. 2: 488. 1766.

Type: [published illustration] "Quis qualis" in Rumphius, Herb. Amboin. 5: t. 38.1747 (lectotype selected by Maurin et al. (2020: 233).

(=) Quisqualis glabra Burm.f., Fl. Ind.: 104, t. 28, f. 2. 1768.

Type: Java, 1758, Anon. s.n. (G-PREL barcode G00805923, lectotype selected by Maurin et al. (2020: 233).

(=) Quisqualis pubescens Burm.f., Fl. Ind.: 104. 1768.

Type: [published illustration] "Quis qualis" in Rumphius, Herb. Amboin. 5: t. 38.1747 (lectotype selected by Merrill (1917: 390).

(=) Quisqualis ebracteata P.Beauv., Fl. Oware 1: 56, 57, t. 35. 1806.

Type: Oware or Bénin, Palisot de Beauvois s.n. (G barcode G00446747 [2 sheets], lectotype selected by Maurin et al. (2020: 233).

(=) Quisqualis obovata Schumach. \& Thonn., Beskr. Guin. Pl. 218-219. 1827.

Type: Guinea, P. Thonning 315 (C barcode C10004457, lectotype selected by Hepper (1976: 40).

(=) Quisqualis indica Lour., Fl. Cochinchin. 274. 1790, nom. illegit., non Q. indica L. (1762).

- Quisqualis loureiroi G.Don, Gen. Hist. 2: 667. 1832, as 'Loureiri'.

Type: J. de Loureiro s.n. (BM barcode BM000947033, lectotype selected at the first step by Exell 1931: 124, and at the second step by Maurin et al. (2020: 233).

(=) Quisqualis villosa Roxb., Fl. Ind. 2: 426. 1832. - Quisqualis indica var. villosa (Roxb.) Kurz, Forest Fl. Burma 1: 467. 1877.

Type: India, W. Roxburgh s.n. (BR barcode BR000000583537, lectotype selected by Maurin et al. (2020: 233). 
(=) Quisqualis sinensis Lindl., Edwards's Bot. Reg. 30: t. 15. 1844.

Type: [published illustration] 'Quisqualis sinensis' in Lindley, Edwards's Bot. Reg. 30: t. 15. 1844 (lectotype selected by Maurin et al. (2020: 233).

(=) Quisqualis spinosa Blanco, Fl. Filip., ed. 2: 254, 1845. Type: Philippines, Palawan, Taytay, 9 May 1913, Merrill: Species Blanconae No. 582 (US barcode US00623716, neotype selected by Maurin et al. (2020: 233); isoneotypes $\mathrm{BM}, \mathrm{K})$.

(=) Quisqualis longiflora C.Presl, Epimel. Bot. 216. 1851. Type: Martaban Helfer (not traced).

(=) Quisqualis grandiflora Miq., J. Bot. Neerl. 1: 119. 1861.

Type: China austr., R. Krone 146 (U barcode U.1217341, lectotype selected by Maurin et al. (2020: 233).

(=) Quisqualis indica var. oxypetala Kurz, Forest Fl. Burma 1: 467. 1877.

Type: Burma, Ava, Kakhyen Hills (not traced).

1.6 Combretum langkawiense O.Maurin \& Christenh., Phytotaxa 451: 233. 2020

Replaced synonym: Quisqualis densiflora var. parvifolia Ridl., Fl. Malay Penins. 1: 711. 1922.

Type: Peninsular Malaysia, Langkawi, Dayang Bunting, November 1916, H.C. Robinson 6198 (K barcode K000786137, lectotype selected by Maurin et al. (2020: 233); isolectotype BM barcode BM000947036 (fragment ex K).

(झ) Quisqualis parvifolia (Ridl.) Exell, J. Bot. 69: 123. 1931.

1.7 Combretum latifolium Blume, Bijdr. (13): 641. 1826

Type: C.L. Blume s.n. (NY barcode NY00245959, lectotype designated at the first step by Gangopadhyay and Chakrabarty (1997: 311), and at the second step here).

(झ) Embryogonia latifolia (Blume) Blume, Mus. Bot. 2: 122. 1856.

(=) Combretum extensum Roxb. ex G.Don, Trans. Linn. Soc. 15: 422.1827.

Type: India orientalis, W. Roxburgh s.n. (G barcode G00446751, lectotype designated here).
(=) Combretum extensum Roxb., Fl. Ind. 2: 229. 1832, nom. illegit., non C. extensum Roxb. ex G.Don (1827).

Type: [unpublished illustration] Icones Roxburghianae No. 1830 (K, lectotype designated here).

(=) Combretum macrophyllum Roxb., Fl. Ind. 2: 231. 1832.

Type: not traced.

(=) Combretum rotundifolium Roxb., Fl. Ind. 2: 226-227. 1832, nom. illegit., non. C. rotundifolium Rich. (1792). Combretum cyclophyllum Steud., Nomencl. Bot., ed. 2, 1: 400. 1840.

Type: W. Roxburgh s.n. (BM barcode BM01382528, lectotype designated here).

(=) Combretum wightianum Wall. ex Wight \& Arn., Prodr. Fl. Ind. Orient. 316. 1834.

Type: EIC 4007 (CAL, lectotype selected by Gangopadhyay and Chakrabarty (1997: 299).

(=) Combretum formosum Griff., Not. Pl. Asiat. 4: 682. 1854, nom. illegit., non C. formosum G.Don (1827).

Type: Burma, Mergue, in sylvis Kulweng, Dec 1834 (not traced).

(=) Combretum horsfieldii Miq., Fl. Ned. Ind. 1(1): 609. 1856.

Type: Java, Horsfield s.n. (holotype $\mathrm{K}$ barcode K0011297290).

(=) Combretum latifolium var. ellipticum Miq., Fl. Ned. Ind. 1(1): 609. 1856, as 'elliptica'.

Type: Java, Horsfield s.n. (holotype K barcode K001129730).

(=) Combretum leucanthum Van Heurck \& Müll.Arg., Observ. Bot. Descript. Pl. Nov. Herb. Van Heurckiani 2: 240. 1871.

Type: Ceylon, Thwaites C.P. 1601 (syntypes BR, G).

(=) Combretum platyphyllum Van Heurck \& Müll.Arg., Observ. Bot. Descript. Pl. Nov. Herb. Van Heurckiani 2: 242. 1871.

Type: EIC 3995 (lectotype G, selected by Gangopadhyay and Chakrabarty (1997: 299).

Notes

In the protologue for Combretum latifolium Blume gave the locality 'in sylvis montosis Salak'. I have failed to locate any Blume specimens that mention Mt Salak, so follow Gangopadhyay and Chakrabarty (1997: 311) 
who selected unlocalised Blume material in NY as the type. As there are actually two specimens present in NY, a second-stage lectotypification is made here. Gangopadhyay and Chakrabarty (1997: 311) designated a Horsfield specimen in CAL as lectotype for C. horsfieldii, but the set of Horsfield specimens seen by Miquel when working on Flora van Nederlandsch Indië are in Kew and the single specimen present is therefore the holotype of Miquel's name.

1.8 Combretum nigrescens King, J. Asiat. Soc. Bengal, Pt. 2, Nat. Hist. 66: 340. 1897

Type: Peninsular Malaysia, Perak, October 1882, Dr King's Collector 3469 (K barcode K000786600, lectotype designated here; isolectotype $\mathrm{BM}$ ).

(=) Combretum kunstleri King, J. Asiat. Soc. Bengal, Pt. 2, Nat. Hist. 66: 340. 1897. - Combretum nigrescens var. kunstleri (King) Ridl., Fl. Malay. Penins. 1: 710. 1922.

Type: Peninsular Malaysia, Perak, Larut, October 1884, Dr King's Collector 6664 (K barcode K000786597, lectotype designated here; isolectotype $\mathrm{BM}$ ).

(=) Combretum scortechinii King, J. Asiat. Soc. Bengal, Pt. 2, Nat. Hist. 66: 341. 1897.

Type: Peninsular Malaysia, Perak, Goping, April 1885, Fr. B. Scortechini 1942 (K barcode K000786602, lectotype designated here).

(=) Combretum glandulosum Slooten, Bijdr. Combret. Flacourt. Ned.-Ind. 39. 1919, nom. illegit., non C. glandulosum F.Hoffm. (1889). - Combretum adenophorum Slooten, Bull. Jard. Bot. Buitenzorg, sér. 3, 6: 56. 1924.

Type: Sumatra, 1880 H. Forbes 3100 (L barcode L.2500736, lectotype designated here; isolectotypes BM barcode BM000947044, L barcode L.2500737).

(=) Combretum elmeri Merr., Univ. Calif. Publ. Bot. 15: 214. 1929.

Type: Borneo, British North Borneo [Sabah], Elphinstone Province, Tawao [Tawau], October 1922-March 1923, A.D.E. Elmer 21440 (UC barcode UC290634, lectotype designated here; isolectotypes A barcode A00068596, BISH barcode BISH1001094, BM(2 sheets), BR barcode BR0000005629534, C barcode C10009529, CAS barcode CAS0033025, F barcode F0054644F, GH barcode GH00068595, HBG barcode HBG514719, K barcode K000786582, L barcode L0009752, M barcode M0146601, MO barcode MO-176152, MSC barcode MSC0091506, NY barcode NY00245957, PH barcode PH00006505, S sheet no. S09-25745, SINGx2 barcode SING0055423, U barcode U0226823, US(2 sheets) barcodes US00810961 US00810960, Z barcode Z-000002869).

(=) Combretum elmeri Merr. var. glabrescens Merr., Univ. Calif. Publ. Bot. 15: 215. 1929.

Type: Borneo, British North Borneo [Sabah], Elphinstone Province, Tawao [Tawau], October 1922-March 1923, A.D.E. Elmer 21561 (UC barcode UC290635, lectotype designated here; isolectotypes A barcode A00068598, BISH barcode BISH1001095, BR barcode BR0000005629206, C barcode C10009531, CAS barcode CAS0032976, GH barcode GH00068597, HBG barcode HBG514718, K barcode K000786581, L barcode L0009753, M barcode M0146600, MICH barcode MICH1192150, MIN barcode MIN1002830, NY barcode NY00245958, S sheet no. S09-25747, SING barcode SING0055422, U barcode U0226815, US barcode US00117555. Z barcode Z-000002870).

(=) Combretum kunstleri var. rectinerve Craib, Fl. Siam. Enum. 1: 617. 1931.

Type: Thailand, Pattani, Bukit, A.F.G. Kerr 7126 (K barcode K000786588, lectotype designated here; isolectotypes ABD, BK barcode BK257790, BM barcode BM000947045).

1.9 Combretum porterianum (C.B.Clarke) Wall. ex Craib., Fl. Siam. Enum. 1(4): 618. 1931

Bas.: Combretum chinense var. porterianum C.B.Clarke in J.D.Hooker, Fl. Brit. India 2: 457. 1878.

(झ) Combretum wallichii DC. var. porterianum (C.B.Clarke) M.Gangop. \& Chakrab., J. Econ. Taxon. Bot. 17: 681. 1993.

Type: EIC 4000 (CAL, lectotype selected by Gangopadhyay and Chakrabarty 1997: 324; isolectotypes K barcode K000786603, K-W barcode K001117875, M M0146610).

\subsection{Combretum punctatum Blume, Bijdr. 640. 1826}

Type: Java, G. Parang, C.L. Blume s.n. (L barcode L0844301, lectotype designated here).

Notes

In the protologue, Blume referred to 'in montosis Parang, et ad pedem montium Salak, Tjerimai etc'. Gangopadhyay and Chakrabarty (1997) designated a Blume 
specimen in NY as the lectotype, but it is not annotated with any of the locations mentioned by Blume. There are specimens with these localities stated in L. Therefore, a lectotype is here designated from one of the specimens in $\mathrm{L}$ to supersede the designation of Gangopadhyay and Chakrabarty.

\subsection{0a Combretum punctatum var. punctatum}

1.10b Combretum punctatum var. squamosum (Roxb. ex G.Don) M.Gangop. \& Chakrab., J. Econ. Taxon. Bot. 17: 680.1993

Bas.: Combretum squamosum Roxb. ex G.Don, Trans. Linn. Soc. 15: 419, 438. 1827.

Type: Roxburgh in herb. Linn. Soc. (not traced).

(झ) Poivrea squamosa (Roxb. ex G.Don) Walp., Repert. Bot. Syst. 2: 64. 1843.

(झ) Combretum punctatum subsp. squamosum (Roxb. ex G.Don) Exell, Fl. Males. ser. 1 4: 539. 1954.

(=) Combretum squamosum Roxb., Fl. Ind. 2: 231. 1832, nom. illegit., non C. squamosum Roxb. ex G.Don.

(=) Combretum distillatorium Blanco, Fl. Filip. 295. 1837. Type: Philippines, Luzon, Bulacan, Maon River, Merrill: Species Blancoanae 847 [M. Ramos leg.] (US barcode US00623715, neotype designated here; isoneotype BM).

(=) Combretum lepidotum C.Presl, Abh. Königl. Böhm. Ges. Wiss., ser. 5, 3: 572. 1845

Type: ?Burma, J.W. Helfer 116 (PR sheet no. 615825, lectotype designated here).

(=) Combretum squamosum var. luzonicum C.Presl, Abh. Königl. Böhm. Ges. Wiss., ser. 5, 6: 576. 1851.

Type: Philippines, H. Cuming 1122 (syntype BM).

(=) Combretum squamosum var. aureum C.B. Clarke in Hooker, Fl. Brit. Ind. 2: 456. 1878.

Type: Burma, Prome Hills, 1827, N. Wallich 252 [EIC 3988[A] p.p.] (K barcode K0011178381, lectotype designated at the first step by Gangopadhyay and Chakrabarty (1997: 314) and at the second step here).

(=) Combretum foliatum Craib, Bull. Misc. Inform. Kew 1930: 163. 1930.

Type: Thailand, Puket, Pang-nga, Nop Pring, 25 February 1929, A.F.G. Kerr 17239 (K (2 sheets) barcodes
K000786592, K000786593, lectotype designated here; isolectotypes $\mathrm{BK}$ barcode BK257797, BM barcode BM000947046).

(=) Combretum squamosum var. dissitum Craib, Flor. Siam. Enumerat. 1: 620. 1931.

Type: Thailand, Maharat, Lampang, Me Tia, 28 April 1923, Winit 860 (K barcode K001129728, lectotype designated here).

Notes

I prefer to recognise the infraspecific taxa of Combretum punctatum at the rank of variety following Gangopadhyay and Chakrabarty (1997) and Chen and Turland (2007), rather than subspecies (Exell 1954).

1.11 Combretum quadrangulare Kurz, J. Asiat. Soc. Bengal, Pt. 2, Nat. Hist. 43(3): 188. 1874

Type: Burma, Tenasserim, Helfer s.n. [Kew distrib. no. 2181] (lectotype CAL, selected by Gangopadhyay and Chakrabarty (1997: 317).

(=) Combretum quadrangulare var. lanceolatum Gagnep., Fl. Gen. 2: 746. 1920.

Type: Cambodia, Prèy-kdey, Kg Speu, 25 April 1909, Magnen et al. s.n. [Châtillon leg.] (P barcode P05046162, lectotype designated here).

1.12 Combretum roxburghii Spreng., Syst. Veg. 2: 231. 1825

Replaced synonym: Combretum decandrum Roxb., Pl. Coromandel 1: 43, t. 59. 1796, nom. illegit., non $C$. decandrum Jacq. (1760).

Type: W. Roxburgh s.n. [EIC 4009[A]] (K-W barcode K001117895, lectotype designated here).

(三) Poivrea roxburghii (Spreng.) DC., Prodr. 3: 18. 1828.

(三) Pentaptera roxburghii (Spreng.) Tul., Ann. Sci. Nat., Sér. 4 6: 84. 1856.

(=) Combretum klossii Ridl., J. Fed. Malay States Mus. 10: 90. 1920.

Type: Thailand, Koh Pipidon [Ko Phi Phi Don], C.B. Kloss 6556 (K barcode K000786591, lectotype designated here; isolectotype SING barcode SING0055423). 
Notes

Roxburgh's name was an illegitimate later homonym when it was published. Gangopadhyay and Chakrabarty (1997) took up Combretum album Pers. as the correct name. This appears in parentheses after C. decandrum in Persoon's Synopsis plantarum (Persoon 1805). The nature of Persoon's parenthetical names is controversial. In the Preface (p. ix), Persoon wrote 'E contrario in parenthesi nomen quasi secundarium passim addidi, quo idea plantae forte distinctior concipi possit.' It is difficult to equate Persoon's 'quasisecondary' names with replacement or alternative names as currently employed, so Combretum album Pers. is not considered validly published. Combretum roxburghii is therefore the earliest replacement name for C. decandrum Roxb.

1.13 Combretum sundaicum Miq., Fl. Ned. Ind., Eerste Bijv. 327. 1861

Type: Sumatra, prov. Lampong, Tiga-nennin, HB 4330 (U barcode U0001193, lectotype designated here).

(=) Combretum oliviforme A.C.Chao, Acta Phytotax. Sin. 7: 244. 1958, 'olivaeforme'.

Type: China, Hainan, Bak Sa, 23 July 1936, S.K. Lau 27571 (holotype IBSC barcode IBSC0004026; isotypes A barcode A00244267, IBK barcode IBK00127780, NAS barcode NAS00047141, PE barcode PE00994471.

(=) Combretum oliviforme A.C.Chao var. yaxianense Y.R.Ling, Acta Phytotax. Sin. 19: 388. 1981.

Type: China, Hainan, [Ya Xian], 11 August 1933, C. Wang 33616 (holotype IBSC barcode IBSC0004027; isotype A barcode A00068593).

1.14 Combretum tetralophum C.B.Clarke in Hooker, Fl. Brit. India 2: 454. 1878

Type: Peninsular Malaysia, Malacca, 1845, W. Griffith s.n. (K barcode K000786605, lectotype selected by Lecompte (1969: 49).

(झ) Combretum tetragonocarpum var. tetralophum (C.B.Clarke) M.Gangop. \& Chakrab., J. Econ. Taxon. Bot. 17: 697. 1993.

(=) Combretum wrayi King, J. Asiat. Soc. Bengal, Pt. 2, Nat. Hist. 66: 339. 1897.

Type: Peninsular Malaysia, Perak, Matang, July 1888, $L$. Wray 2504 (K barcode K000786607, lectotype designated here; isolectotype BM barcode BM000947040).
1.15 Combretum trifoliatum Vent., Choix Pl. t. 58. 1808

Type: Java, La Haye s.n. (G barcode G00177911, lectotype selected by Lecompte (1969: 40).

(झ) Cacoucia trifoliata (Vent.) DC., Prodr. 3: 22. 1828.

(=) Combretum lucidum Blume, Bijdr. Fl. Ned. Ind. 641. 1826. - Cacoucia lucida (Blume) Hassk., Flora 27: 607. 1844. - Embryogonia lucida (Blume) Blume, Mus. Bot. Lugd.-Bat. 2: 122. 1856.

Type: Java, Pamanoekang, C.L. Blume 1543 (L barcode L0844262, lectotype designated here).

(=) Terminalia lancifolia Griff., Not. Pl. Asiat. 4: 685. 1854.

Type: Moulmein ad marginem sylvarum (not traced).

2. Getonia Roxb., Pl. Coromandel 1: 61, t. 87. 1798.

Type: Getonia floribunda Roxb.

(三) Calycopteris Poir., Encycl., Suppl. 2: 41. 1811, nom. illegit. superfl.

Notes

Chen and Turland (2007: 315) explained why Calycopteris Lam. was not validly published and hence Getonia is the correct name for the genus.

2.1 Getonia floribunda Roxb., Pl. Coromandel 1: 61, t. 87. 1798

Type: [published illustration] Roxburgh, Pl. Coromandel 1: t. 87.1798 (lectotype designated here).

(झ) Calycopteris floribunda (Roxb.) Lam. ex Poir., Encycl. Suppl. 2: 41. 1811.

(=) Getonia nitida Roth, Nov. Pl. Sp. 217. 1821. - Getonia nutans Roxb. ex DC., Prodr. 3: 15. 1828, nom. illegit. superfl. - Calycopteris nutans Kurz, J. Asiat. Soc. Bengal, Pt. 2, Nat. Hist. 46(2): 59. 1877, nom. illegit. superfl.

Type: B. Heyne s.n. [EIC 4013A] (K-W barcode K001117915, neotype designated here, excluding material on sheet annotated ' $c$ ' in pencil).

(=) Getonia nutans var. brachystachya DC., Prodr. 3: 15. 1828.

Type: ex herb. Thibaud (holotype G-DC IDC800 475/24). 
(=) Getonia nutans Roxb., Fl. Ind. 2: 428. 1832, nom. illegit., non G. nutans Roxb. ex DC. (1828)

Type: W. Roxburgh s.n. [EIC 4012[A]] (K-W barcode K001117911, lectotype designated here excluding material labelled ' $\mathrm{B}$ ' in pencil on the sheet).

(=) Calycopteris nutans Kurz var. roxburghii Kurz, J. Asiat. Soc. Bengal, Pt. 2, Nat. Hist. 46(2): 59. 1877.

Type: not traced.

(=) Calycopteris nutans Kurz var. glabriuscula Kurz, J. Asiat. Soc. Bengal, Pt. 2, Nat. Hist. 46(2): 59. 1877.

Type: not traced.

Notes

Gangopadhyay and Chakrabarty (1997: 296) referred to both the published plate (t. 87) and the unpublished drawing as the type of Getonia floribunda. As both elements are original material, the typification is not effective. A new lectotypification to the published plate is therefore made here. There are some Roxburgh specimens extant, but as this name was published early in Roxburgh's career, it is difficult to be certain that collections were made before the publication date as Roxburgh very rarely provided dates (or much other information) on his specimens.

3. Lumnitzera Willd., Neue Schriften Ges. Naturf. Freunde Berlin 4: 186-187. 1803

Type: Lumnitzera racemosa Willd.

(=) Pyrrhanthus Jack, Malayan Misc. 2(7): 57. 1822.

Type: Pyrrhanthus littoreus Jack

(=) Problastes Reinw., Syll. Pl. Nov. 2: 10. 1825 ['1828'].

Type: Problastes cuneifolia Reinw.

(=) Pokornya Montrouz., Mem. Acad. Lyon 10: 201. 1860. Type: Pokornya ettingshausenii Montrouz.

\section{Key to Lumnitzera species}

1. Inflorescences terminal, flowers pedicellate, petals red; stamens at least $\times 2$ as long as erect petals 3.1 L. littorea

Inflorescences axillary; flowers more or less sessile, petals white, stamens scarcely exceeding length of erect petals..... 3.2. L. racemosa
3.1 Lumnitzera littorea (Jack) Voigt, Hort. Suburb. Calcutt. 39.1845

Bas.: Pyrrhanthus littoreus Jack, Malayan Misc. 2(7): 57. 1822.

Type: Sumatra, W. Jack s.n. (E barcode E00273921, lectotype designated here).

(झ) Lumnitzera coccinea Wight \& Arn., Prodr. Fl. Ind. Orient. 316. 1834, nom. illegit. superfl.

(三) Bruguiera littorea (Jack) Steud., Nomencl. Bot., ed. 2, 1: 231.1840.

(=) Laguncularia coccinea Gaudich., Voy. Uranie, Bot. (2): 68.1826.

Type: Marianas, C. Gaudichaud-Beaupré s.n. (P barcode P01901322, lectotype designated here).

(=) Laguncularia purpurea Gaudich., Voy. Uranie, Bot. (12): 481. 1830. - Lumnitzera purpurea (Gaudich.) C.Presl, Repert. Bot. Syst. 1: 155. 1833.

Type: Marianas, C. Gaudichaud-Beaupré s.n. (P barcode P01901322, lectotype designated here).

(=) Lumnitzera pedicellata C.Presl, Reliq. Haenk. 2: 23. 1830. - Laguncularia haenkei Endl., Ann. Wiener Mus. Naturgesch. 1: 180. 1836, nom. illegit. superfl. - Laguncularia pedicellata (C.Presl) Steud., Nomencl. Bot., ed. 2, 2: 5.1840.

Type: Marianas, T.P.X. Haenke s.n. (PRC barcode PRC450418 lectotype designated here; isolectotypes HAL barcode HAL118786, LE barcode LE00016815, PR sheet nos. 25245a, 25245b).

(=) Petaloma coccineum Blanco, Fl. Filip. 345. 1837, as 'coccinea'.

Type: Philippines, Luzon, Tayabas Province, Mt Kinatakian, 6 April 1913, Merrill: Species Blancoanae No. 521 [L. Escritor leg.] (US barcode US 00623717, neotype designated here; isoneotypes BM, K barcode K001129749).

(=) Lumnitzera pentandra Griff., Not. Pl. Asiat. 4: 684. 1854. Icon. Pl. As. T. 644A 1854

Type: Mergue, Inter Rhizophora, Madamacca, Sept 1834, Griffith (not traced).

Notes

Gangopadhyay and Chakrabarty (1997: 326) selected a specimen from K-W under number 4018A (K001117945) as lectotype of Pyrrhanthus littoreus Jack. However, this specimen was collected by Wallich from 
Pulau Dinding in 1822 . It seems very unlikely Jack saw the specimen, but the typification would stand as a neotypification. However, there is a Jack specimen clearly labelled in E. This is here designated as lectotype to supersede the earlier designation.

Laguncularia coccinea Gaudich. was first validated in part 2 of the Voyage ... l'Uranie rather than part 11 as generally cited: "une combrétacée du genre Laguncularia ( $L$. coccinea), planche 104, et qui est le kada-kandel de Rheed. Mal. 6, planche 37, à fleurs rouge-ponceau trèsvif, désigné ici sous le nom de guia-guia." The Rheede element actually refers to the white-flowered Lumnitzera racemosa, but Gaudichaud-Beauprés mention of "very bright poppy-red flowers" seems sufficient to distinguish the species as this is a highly distinctive character. There also seems to have been confusion over the chosen epithet with $L$. purpurea being used in part 12, though apparently referring to the same taxon. These two names are lectotypified to the same specimen from Guam here.

3.2 Lumnitzera racemosa Willd., Neue Schriften Ges. Naturf. Freunde Berlin 4: 187. 1803

Type: India, J.G. Klein s.n. (B-W barcode B-W08141-010, lectotype selected by Wickens (1973: 93); isolectotype DNA (fragment).

(=) Funckia karakandel Dennst., Schlüssel Hortus Malab. 32. 1818.

Type: [published illustration] Rheede, Hort. Malab. 6: t. 37.1685 (lectotype selected by Gangopadhyay and Chakrabarty (1997: 326).

(=) Problastes cuneifolia Reinw., Syll. Pl. Nov. 2: 10. 1828. Type: [published illustration] Rheede, Hort. Malab. 6: t. 37. 1685 (lectotype selected by Gangopadhyay and Chakrabarty (1997: 329).

(=) Bruguiera madagascariensis DC., Prodr. 3: 28. 1828.

Type: [published illustration] Rheede, Hort. Malab. 6: t. 37.1685 (lectotype selected by Gangopadhyay and Chakrabarty (1997: 326).

(=) Petaloma alternifolium Roxb., Fl. Ind. 2: 373. 1832, as 'alternifolia'.

Type: [published illustration] Rheede, Hort. Malab. 6: t. 37. 1685 (lectotype selected by Gangopadhyay and Chakrabarty (1997: 329).

(=) Petaloma album Blanco, Fl. Filip. 344. 1837, as alba. Type: Philippines, Luzon, Province of Rizal, Maricaban,
March 1914, Merrill: Species Blancoanae No. 303 (US barcode US00623718, neotype designated here; isoneotypes BM, K barcode K001129748).

(=) Pokornya ettingshausenii Montrouz., Mem. Acad. Lyon 10: 201. 1860, 'Ettingshanseni'.

Type: Ile d'Art, Montrouzier (not traced).

(=) Lumnitzera edulis Blume ex Laness., Pl. Util. Col. Franç.: 256. 1886.

Type: New Caledonia, Marais de Gatope, 1861-1867, E. Vieillard 2571 (K barcode K001129747, lectotype designated here).

(=) Lumnitzera racemosa var. pubescens Koord. \& Valet., Meded. Lands Planten. 61: 33, 34. 1903

Type: Java, Ins. Karimon, Koorders (not traced).

4.1 Terminalia L., Syst. Nat. (ed. 12) 2: 665, 674. 1767, nom. cons.

Type: Terminalia catappa L.

(=) Buceras P.Browne, Civ. Nat. Hist. Jamaica 221. 1756, nom. rejic.

Type: Bucida buceras L.

(=) Bucida L., Syst. Nat. (ed. 10) 2: 1025, 1368. 1759, nom. cons. (against Buceras P.Browne) and nom. rejic. (against Terminalia L.).

Type: Bucida buceras L.

(=) Adamaram Adans., Fam. Pl. 2: 445. 1763, nom. rejic.

Type: Terminalia catappa L. (neotype selected by Exell (1931: 125).

(=) Myrobalanifera Houtt., Nat. Hist. 2(2): 485, t. 10, f. 2. 1774.

Type: Myrobalanifera citrina Houtt.

(=) Pamea Aubl., Hist. Pl. Guiane 946, t. 359. 1775, nom. rejic. (against Buchenavia Eichler).

Type: Pamea guianensis Aubl.

(=) Tanibouca Aubl., Hist. Pl. Guiane 448. 1775.

Type: Tanibouca guianensis Aubl.

(=) Kniphofia Scop., Intr. Hist. Nat.: 327. 1777, nom. rejic. (against Kniphofia Moench).

Type: Terminalia catappa L. (neotype designated here). 
(=) Chuncoa Pav. in Juss., Gen. Pl. 76. 1789. - Gimbernatia Ruiz \& Pav., Fl. Peruv. Prodr. 138, t. 36. 1794, nom. illegit. superfl.

Type: Chuncoa amazonia J.F. Gmel.

(=) Badamia Gaertn., Fruct. Sem. Pl. 2: 90. 1790.

Type: Badamia commersonii Gaertn.

(=) Myrobalanus Gaertn., Fruct. Sem. Pl. 2: 90. 1790. Terminalia section Myrobalanus (Gaertn.) DC., Prodr. 3: 12. 1828.

Type: Myrobalanus bellirica Gaertn. (lectotype selected by Pfeiffer (1874: 394).

(=) Catappa Gaertn., Fruct. Sem. Pl. 2: 206. 1791. - Terminalia sect. Catappa (Gaertn.) DC., Prodr. 3: 10. 1828. Type: Catappa benzoin Gaertn.

(=) Hudsonia A. Rob. ex Lunan, Hort. Jamaic. 2: 310. 1814, nom. illegit., non Hudsonia L. (1767).

Type: Hudsonia arborea A.Rob. ex Lunan.

(=) Fatraea Juss., Dict. Sc. Nat. 16: 206. 1820.

Type: Fatraea buxifolia Juss.

(=) Ramatuela Kunth, Nov. Gen. Sp. 7: 253, t. 656. 1825.

- Terminalia sect. Ramatuela (Kunth) Alwan \& Stace, Ann. Missouri Bot. Gard. 76(4): 1126. 1989, 'Ramatuella'. Type: Ramatuela argentea Kunth

(=) Conocarpus sect. Anogeissus DC., Prodr. 3: 16. 1828. - Anogeissus (DC.) Wall. ex Guill. \& al., Flor. Seneg. Tent. 1: 279. 1832.

Type: Conocarpus acuminatus Roxb. ex DC.

(=) Pentaptera Roxb. ex DC., Prodr. 3: 14. 1828.

Type: Pentaptera arjuna Roxb. ex DC. (lectotype designated here).

(=) Vicentia Allemão, Pl. Novas Brasil cum tab. 1844.

Type: Vicentia acuminata Allemão

(=) Chicharronia A.Rich., Hist. Phys. Cuba, Pl. Vasc. 529, t. 43. 1845. - Terminalia sect. Chicharronia (A.Rich.) Alwan \& Stace, Fl. Neotrop. Monogr. 107: 187. 2010. Type: Chicharronia intermedia A.Rich.

(=) Buchenavia Eichler, Flora 49(11): 164. 1866, nom. cons.

Type: Bucida capitata Vahl (lectotype selected by Stace (2007: 41).
(=) Pteleopsis Engl., Abh. Königl. Akad. Wiss. Berlin 1894: 25. 1894.

Type: Pteleopsis variifolia Engl.

(=) Finetia Gagnep., Notul. Syst. (Paris) 3: 278. 1916.

Type: Finetia rivularis Gagnep.

(=) Terminaliopsis Danguy in Lecomte et al., Madag. Bois Analamaz. 110. 1922; Bull. Mus. Natl. Hist. Nat. 29(1): 108. 1923.

Type: Terminaliopsis tetrandrus Danguy

\section{Key to Terminalia species}

1. Leaves spirally arranged, clustered at swollen ends of twigs

Leaves generally opposite to subopposite, not clustered at twig ends, twig ends not notably swollen ... 8

2. Leaves generally exceeding $10 \mathrm{~cm}$ wide; petiole to $12 \mathrm{~mm}$ long...... 4.3 T. catappa

Leaves rarely exceeding $10 \mathrm{~cm}$ wide; petiole more than 12 $\mathrm{mm}$ long

3. Leaves with apex obtuse to acuminate 4

Leaves with apex rounded to emarginate. 7

4. Leaves with venation distinctly raised above in dry leaves; fruits without wings, densely velvety pubescent. 4.1 T. bellirica

Leaves with venation more or less flush to sunken above in dry leaves; fruits winged or if not winged then glabrous to tomentose, but not densely velvety pubescent 5

5. Petioles not exceeding $2.5 \mathrm{~cm}$ long; fruits fleshy without wings. 4.6 T. foetidissima

Petioles generally more than $2.5 \mathrm{~cm}$ long; fruits dry with 2 wings.

6. Leaves glaucous below; fruits densely hairy with wings about as wide as long. 4.7 T. glaucifolia

Leaves not glaucous below; fruits sparsely hairy with wings distinctly wider than long 4.2 T. calamansanai

7. Leaves glaucous below; petioles $3 \mathrm{~cm}$ long or more; fruits winged 4.10 T. subspathulata

Leaves not glaucous below; petioles to $2.5 \mathrm{~cm}$ long; fruits not winged 4.9 T. phellocarpa

8. Inflorescences axillary spikes; fruits generally with 2 wings .. 4.8 T. mucronata 9 
9. Leaves with pair of glands on lamina margin near base rather than on petiole; fruits with three wings 4.11 T. triptera

Leaves with pair of glands generally visible on petiole rather than lamina; fruits without wings ..... 10

10. Leaves to $6 \mathrm{~cm}$ wide, glabrous; fruits ellipsoid to fusiform apex beaked, endocarp with 5-angled sides..... 4.5 T. citrina

Leaves generally more than $6 \mathrm{~cm}$ wide, hairy below; fruits globose to ovoid, apex rounded, not beaked, endocarp more or less round in cross section, not 5-angled. 4.4 T. chebula

4.1 Terminalia bellirica (Gaertn.) Roxb., Pl. Coromandel 2: 54, t. 198. 1805, as 'bellerica'

Bas.: Myrobalanus bellirica Gaertn., Fruct. Sem. Pl. 2: 90, t. 97 , f. 2.1790.

Type: [published illustration] Gaertner, Fruct. Sem. Pl. 2: t. 97, f. 2. 1790 (lectotype selected by Wickens (1990: 7).

(三) Terminalia myrobalana B.Heyne ex Roth, Nov. Pl. Sp. 378. 1821, nom. illegit. superfl.

(=) Myrobalanus taria Buch.-Ham., Trans Linn. Soc. London 17: 159. 1835 ['1837']. - Terminalia taria Buch.Ham., Trans Linn. Soc. London 17: 159. 1835 ['1837']. Type: India, Mysore, F. Buchanan-Hamilton s.n. (BM, lectotype designated here).

(=) Myrobalanus tania Buch.-Ham. ex Kostel., Allg. Med.-Pharm. Fl. 4: 1497. 1835.

Type: [published illustration] 'Tani', Rheede, Hort. Malab. 4: t. 10. 1683 (lectotype designated here).

(=) Terminalia punctata Roth, Nov. Pl. Sp.: 381. 1821. Type: J.P. Rottler s.n. (K barcode K000786123, neotype designated here).

(=) Terminalia attenuata Edgew., Trans. Linn. Soc. London 20: 46. 1846.

Type: India, Himalaya, altit. 4000 ped., near Jugutgurh, M.P. Edgeworth 6 (K barcode K000786121, lectotype designated here).

(=) Terminalia gella Dalzell, Hooker's J. Bot. Kew Gard. Misc. 3: 227. 1851, as Gella.

Type: Crescit rar in Concano australiore (not traced).

(=) Terminalia laurinoides Teijsm. \& Binnend. in Miquel, Fl. Ned. Ind. 1: 600. 1856. - Terminalia bellirica var. laurinoides (Teijsm. \& Binnend.) C.B.Clarke in Hooker, Fl. Brit. India 2: 445. 1878. - Myrobalanus laurinoides (Teijsm. \& Binnend.) Kuntze, Revis. Gen. Pl. 1: 237. 1891. Type: Java, J.E. Teijsmann s.n. (L barcode L0843169, lectotype designated here).

4.2 Terminalia calamansanai (Blanco) Rolfe, J. Linn. Soc., Bot. 21: 310. 1884

Bas.: Gimbernatia calamansanai Blanco, Fl. Filip., ed. 2 266. 1845.

Type: Philippines, Mindanao, Butuan Subprovince, October 1913, D.P. Miranda For. Bur. 20781 [Merrill: Species Blancoanae No. 605 p.p. (fruiting material only)] (US barcode US00623709, neotype designated here excluding flowering material mounted on the same sheet).

(=) Pentaptera pyrifolia C.Presl, Epimel. Bot. 215. 1851. - Terminalia pyrifolia (C.Presl) Kurz, Prelim. Rep. For. Veg. Pegu App. A: lix. 1875.

Type: Moulmein, J.W. Helfer (not traced).

(=) Terminalia papilio Hance, J. Bot. 15: 333. 1877.

Type: Vietnam, Ins. Phu Kok, L. Pierre s.n. [Herb. Hance 19745] (BM, lectotype designated here).

(=) Terminalia bialata var. cuneifolia C.B.Clarke in Hook.f., Fl. Brit. India 2: 449. 1878.

Type: Burma, Ava, 22 October [1826], N. Wallich 315 [EIC 3972, p.p.] (K-W barcode K001117791, lectotype designated here).

(=) Terminalia blancoi Merr., Philipp. J. Sci., C 4: 645. 1909.

Type: Philippines, Luzon, Province of Rizal, February 1904, Decades of Philippine Forest Flora No. 2 [Ahern's Collector leg.] (NY barcode NY00245999, lectotype designated here; isolectotype US barcode US00117589).

(=) Terminalia calamansanai var. platypteris Merr., Philipp. J. Sci., C 4: 646. 1909.

Type: Philippines, Luzon, Province of Zambales, Mar 1904, W.M. Maule For. Bur. 371 (NY barcode NY00246001, lectotype designated here; isolectotype US barcode US00117606).

(=) Terminalia calamansanai var. acuminata Merr., Philipp. J. Sci., C 4: 646. 1909.

Type: Philippines, Luzon, Prov. Principe, Baler, August-October 1903, E.D. Merrill 1067 (NY barcode 
NY00246000, lectotype designated here; isolectotype US barcode US00117607).

(=) Terminalia oryzetorum Craib, Bull. Misc. Inform. Kew 1928: 69. 1928.

Type: Thailand, Mûang Pichit, 25 March 1922, A.F.G. Kerr 5671 (K barcode K000786200, lectotype designated here; isolectotypes ABD, BM barcode BM000947099, NY barcode NY00245995, P barcode P02286610).

(=) Terminalia latialata C.T.White, J. Arnold Arbor. 10: 249. 1929.

Type: Papua New Guinea, Lower Vailala River, 15 March 1926, L.J. Brass 1147 (A barcode A00068656, lectotype designated here; isolectotypes $\mathrm{BM}$ barcode BM000947096, BRI, K).

\subsection{Terminalia catappa L., Mant. Pl. 1: 128. 1767}

Type: Anon. s.n. (lectotype LINN 1221.1, selected by Byrnes 1977: 38).

(झ) Juglans catappa (L.) Lour., Fl. Cochinch. 2: 703. 1790.

(झ) Myrobalanus catappa (L.) Kuntze, Revis. Gen. Pl. 1: 237. 1891.

(झ) Buceras catappa (L.) Hitchc., Rep. (Annual) Missouri Bot. Gard. 4: 85. 1893.

(=) Phytolacca javanica Osbeck, Dagb. Ostind. Resa 276. 1757.

Type: Java, Pulau Panaitan, west of Tg. Parat, 3 October 1951, J. van Borssum Waalkes 818 (K (2 sheets) barcode K001129735, K001129736, neotype designated here; isoneotype L L0843090).

(=) Terminalia moluccana Lam., Encycl. 1: 349. 1783.

Type: [published illustration] Rumph., Herb. Amboin. 1: t. 68. 1741 (lectotype selected by Merrill (1917: 390).

(=) Terminalia subcordata Humb. \& Bonpl. ex Willd., Sp. Pl., ed. 4, 4(2): 968. 1806. - Terminalia catappa var. subcordata (Humb. \& Bonpl. ex Willd.) DC., Prodr. 3: 11. 1828

Type: Cuba, Havana, F.W.H.A. Humboldt \& A. Bonpland 1329 (B-W barcode B-W18940000, lectotype designated here; isolectotype P-Bonpl. barcode P00679491).

(=) Terminalia intermedia Bertero ex Spreng., Syst. Veg. 2: 359.1825.

Type: Guadeloupe, 1816-1818, C.G. Bertero s.n. (TO).
(=) Terminalia latifolia Blanco, Fl. Filip. 376. 1837, nom. illegit., non T. latifolia Sw. (1788).

Type: Philippines, Luzon, Pangasinan Prov., Umingan, May 1914, Merrill: Species Blancoanae No. 197 (US barcode US00689474, neotype designated here).

(=) Terminalia paraensis Mart., Flora 24 Beibl. 2: 24.1841. Type: Brazil, Pará, in sylvis Prov. Paraensis, C.F.P. Martius s.n. (M sheet no. M-0146722, lectotype designated here; possible isolectotype M sheet no. M-0146723).

(=) Terminalia catappa var. chlorocarpa Hassk., Tijdschr. Natuurl. Gesch. Physiol. 10: 145. 1843.

Type: non designatus.

(=) Terminalia catappa var. macrocarpa Hassk., Tijdschr. Natuurl. Gesch. Physiol. 10: 145. 1843.

Type: [published illustration] Rumph., Herb. Amboin. 1: t. 68.1741 (lectotype designated here).

(=) Terminalia catappa var. rhodocarpa Hassk., Tijdschr. Natuurl. Gesch. Physiol. 10: 145. 1843.

Type: non designatus.

(=) Terminalia rubrigemmis Tul., Ann. Sci. Nat., Bot. sér. 4, 6: 102.1856.

Type: Madagascar (not traced).

(=) Terminalia catappa var. pubescens Kurz, For. Fl. Brit. Burma 1: 454. 1877.

Type: Andamans, W.S. Kurz (not traced).

(=) Terminalia burmanica King ex Prain, J. Asiat. Soc. Bengal, Pt. 2, Nat. Hist. 73(5): 204. 1904.

Type: Burma, Sajein, August 1891, Abdul Huk s.n. (lectotype CAL accession no. 163973-4, selected by Gangopadhyay and Chakrabarty (1997: 343).

Notes

Phytolacca javanica Osbeck is the oldest name available for this well-known seashore species but its transfer to Terminalia is blocked by T. javanica Miq. Osbeck encountered the species on Nieu Eyland (New Island), now called Pulau Peucang, just off the South-West tip of Java. In the absence of any original material, a specimen collected from Pulau Panaitan quite nearby in the Sunda Straits, is here designated as neotype for Osbeck's name.

A name often cited in synonymy of T. catappa is Badamia commersonii Gaertn. However, the original figure and description, which refer to the fruit and seed alone, do not match T. catappa. The fruit is reported as oblong ellipsoidal with a slight obtuse beak and an 
endocarp with six pronounced longitudinal ridges. It is based on a Commerson collection from Madagascar. The Tubingen herbarium has some material labelled Badamia commersonii from Gaertner's herbarium which consists of fruits of T. catappa that certainly differ from the original description and figure. I am inclined to follow Tulasne (1856: 92) who considered that Badamia commersoni was a species similar to Terminalia exsculpta.

Gangopadhyay and Chakrabarty (1997: 343) designated two plates from Rheede's Hortus Malabaricus as lectotype of Badamia commersonii Gaert., but as Gaertner did not cite Rheede, this cannot represent original material. In order to encourage further work on clarifying the true identity of the Gaertner species, below I designate the Gaertner figure as lectotype of Badamia commersonii.

Poiret published Myrobalanus terminalia, apparently entirely based on a figure in Lamarck's Tableau Encyclopédique. This figure is clearly a reworking of Gaertner's Badamia illustration, but as neither Poiret nor Lamarck referred to Gaertner or his name, Poiret's name has to be considered as nomenclaturally independent.

(=) Badamia commersonii Gaertn., Fruct. 2: 90, t. 97 f. $1(\mathrm{a}-\mathrm{g}) .1790$, as 'Commersoni'.

Type: [published illustration] "Badamia Comersoni", Gaertner, Fruct. 2: t. 97 f. 1(a-g). 1790 (lectotype designated here).

(झ) Terminalia badamia DC., Prodr. 3: 12. 1828, nom. illegit. superfl.

(=) Myrobalanus terminalia Poir. in Lamarck, Encycl., Suppl. 3: 707. 1814, as Mirobolanus.

Type: [published illustration] Lamarck, Tabl. Encycl. 2, 5(1): Pl. 849 f. 2. 1799 (lectotype designated here).

\subsection{Terminalia chebula Retz., Observ. Bot. 5: 31. 1788}

Type: J.G. Koenig s.n. (lectotype LD acc. no. 1638155, selected by Fischer (1932: 57).

(झ) Myrobalanus chebula (Retz.) Gaertn., Fruct. Sem. Pl. 2: 91.1790.

(झ) Buceras chebula (Retz.) A.Lyons, Pl. Nam.: 71. 1900.

(=) Myrobalanifera citrina Houtt., Handl. Pl.-Kruidk. 2: 486 , t. 10 f. 2.1774 .

Type: [published illustration] Houttuyn., Handl. Pl.Kruidk. 2: t. 10, f. 2.1774 (lectotype selected by Turner (2014: 11).
(=) Terminalia reticulata B.Heyne ex Roth, Nov. Pl. Sp.: 381. 1821.

Type: India, Cannanore, February 1852, R. Wight s.n. [Peninsula Indiae Orientalis No. 1012] (K barcode K001129738, neotype designated here).

(=) Terminalia aruta Buch.-Ham. ex G.Don, Gen. Hist. 2: 659. 1832.

Type: Buchanan-Hamilton in herb. Lambert (not traced).

(=) Terminalia gangetica Roxb., Fl. Ind. 2: 437. 1832. - Myrobalanus gangetica (Roxb.) Kostel., Allg. Med.Pharm. Fl. 4: 1497. 1835.

Type: not traced.

(=) Terminalia tomentella Kurz, J. As. Soc. Bengal 42: 80. 1873. - Terminalia chebula var. tomentella (Kurz) C.B.Clarke in Hooker, Fl. Brit. Ind. 2: 446. 1878. Myrobalanus tomentella (Kurz) Kuntze, Revis. Gen. Pl. 1: 237. 1891.

Type: Burma, Kurz (not traced).

(=) Terminalia glandulipetiolata De Wild., Pl. Bequaert. 4(3): 344. 1928.

Type: Congo Belge, Eala, 29 December 1920, A.A.P.J.G. Corbisier-Baland 1630 (BR barcode BR00000887320, lectotype designated here; isolectotypes BR barcode BR00000887353, BR00000887386, S acc. no. S09-25894).

4.5 Terminalia citrina (Gaertn.) Roxb. in Fleming, Asiat. Res. 11: 183. 1810

Bas.: Myrobalanus citrina Gaertn., Fruct. Sem. Pl. 2: 91, t. 97, f. 2 n-s. 1790.

Type Cons.: Bangladesh, Mymensingh District, Gabtulli, 8 July 1872, C.B. Clarke 17257 (K K000608176).

(झ) Terminalia chebula Retz. var. citrina (Gaertn.) Gagnep., Fl. Gen. Indo-Chine 2: 753. 1920.

(=) Bucida comintana Blanco, Fl. Filip. 856. 1837. - Terminalia comintana (Blanco) Merr., Philipp. J. Sci., C 4: 300. 1909.

Type: Philippines, Luzon, Batangas Prov., Mt Macolot, 5 Feb 1915, E.D. Merrill: Species Blancoanae 780 [M. Ramos leg.] (US barcode US00623713, neotype designated here; isoneotype BM).

(=) Embryogonia arborea Teijsm. \& Binnend., J. Bot. Neerl. 1: 365. 1861. - Combretum arboreum (Teijsm. \& Binnend.) Miq., Ann. Mus. Bot. 4: 115. 1868. - Terminalia teysmannii Koord. \& Valeton, Meded. Lands Plan- 
tentuin 61: 20. 1902, nom. illegit. superfl. - Terminalia arborea (Teijsm. \& Binnend.) Koord., Exkursionsfl. Java 2: 671. 1912.

Type: Java (not traced).

(=) Terminalia citrina var. malayana Kurz, J. Asiat. Soc. Bengal, Pt. 2, Nat. Hist. 45: 130. 1876.

Type: India, Ins. Nicobar, Expedition Novara 75 [leg. A. Jelinek 132] (W sheet no. W 0080071, lectotype designated here).

(=) Terminalia manii King, J. Asiat. Soc. Bengal, Pt. 2, Nat. Hist. 66: 329. 1897.

Type: Nicobars, Dr King's Collector 485 (CAL, lectotype selected by Gangopadhyay and Chakrabarty (1997: 348); isolectotypes BM, K barcode K001129734).

(=) Terminalia multiflora Merr., Govt. Lab. Publ. Philipp. 27: 34. 1904.

Type: Philippines, Luzon, Province of Rizal, Bosoboso, July 1903, E.D. Merrill 2796 (K barcode K000786176, lectotype designated here; isolectotypes NY barcode NY00246011, US barcode US00117611).

(=) Terminalia curtisii Ridl., Bull. Misc. Inform. 1931: 449. 1931.

Type: Peninsular Malaysia, Penang, Waterfall, June 1896, C. Curtis 3207 (K barcode K000786161, lectotype designated here; isolectotypes K barcode K000786160, SING barcode SING0055417).

4.6 Terminalia foetidissima Griff., Not. Pl. Asiat. 4: 685. 1854

Type: Burma, Mergui, March 1835, W. Griffith s.n. (syntype CAL).

(झ) Myrobalanus foetidissima (Griff.) Kuntze, Revis. Gen. Pl. 1: 237. 1891.

(=) Terminalia sumatrana Miq., Fl. Ned. Ind., Eerste Bijv. 326. 1861.

Type: Sumatra, Palembang, Toeboean, Ogan oeloe, H.B. 3814 [J.E. Teijsmann leg.] (U barcode U0001196, lectotype designated here; isolectotype L barcode L.2493804).

(=) Terminalia oocarpa Merr., Publ. Bur. Sci. Gov. Lab. 17: 32. 1904, as ovocarpa.

Type: Philippines, Luzon, Province of Bataan, Lamao River, November 1903, P.T. Barnes For. Bur. 67 (K barcode K000786170, lectotype designated here).
(=) Terminalia ellipsoidea Merr.

Type: Philippines, Mindoro, Pinamalayan, May 1903, E.D. Merrill 2148 (K barcode K000786172, lectotype designated here; isolectotype US barcode US00117598).

(=) Terminalia borneensis Sloot., Bijdr. Combret. Flacourt. Ned.-Ind. 14. 1919.

Type: Borneo, O. Beccari P.B. 2593 (K barcode K000786182, lectotype designated here; isolectotype FI-B).

4.7 Terminalia glaucifolia Craib, Bull. Misc. Inform. Kew 1928(2): 68. 1928

Type: Thailand, Doi Sootep, 20 August 1910, A.F.G. Kerr 1328 (BK, lectotype selected by Nanakorn 1985, p. 81; isolectotypes ABD, BM, K barcode K000786198, P).

4.8 Terminalia mucronata Craib \& Hutch., Bull. Misc. Inform. Kew 1909(9): 358. 1909

Type: Thailand, Chiengmai, Doi Sootep, 11 April 1909, A.F.G. Kerr 593 (K (4 sheets) barcodes K000786194, K000786194, K000786195, K000786196, lectotype designated here; isolectotypes BM barcode BM000947102, L barcode L0009802, P barcode P02286601, TCD barcode TCD0016741).

(=) Terminalia corticosa Pierre ex Craib \& Hutch., Bull. Misc. Inform. Kew 1909(9): 358. 1909.

Type: Vietnam, Baria, montibus Dinh, March 1862, L. Pierre 203 (P barcode P02286603, lectotype designated here).

(=) Terminalia thorelii Gagnep., Not. Syst. 3: 287. 1916. Type: Cambodia, Ubon, January anno. 1866-1868, C. Thorel 2824 (P barcode P02286602, lectotype designated here).

(=) Parinari euadenia Kosterm., Bot. Tidsskr. 67: 321. 1973.

Type: Thailand, Chiengmai, Doi Sutep, 15 October 1958, T. Sørensen et al. 5687 (holotype C C10017914).

\section{Notes}

The name Terminalia corticosa first appeared in Lanessan (1886: 315). The descriptive content of the account is slight - a size range of the tree and colour of the wood. This has been considered insufficient to validate the name (Gagnepain 1916: 215, Lecompte 1969: 
88). Craib and Hutchinson described Terminalia mucronata in 1909. As well as a fairly detailed description, they included a diagnosis comparing Terminalia mucronata and $T$. corticosa. This effectively validated $T$. corticosa because the description of T. mucronata was sufficient to validate that name. It is not clear on what Craib and Hutchinson based their concept of T. corticosa as I have not found any flowering specimens in $\mathrm{K}$ or $\mathrm{BM}$ that they are likely to have seen. Possibly they visited Paris or had material on loan. In order to maintain continuity, I designate the specimen chosen by Lecompte (1969) as lectotype of T. corticosa Pierre ex Craib \& Hutch. As Lecompte did not cite Craib and Hutchinson's publication, I here establish T. mucronata Craib \& Hutch. as having priority over T. corticosa Pierre ex Craib \& Hutch.

For Terminalia thorelii, the collection number 2823 was cited in the protologue. I have not traced this number and presume it was an error for 2824 .

4.9 Terminalia phellocarpa King, J. Asiat. Soc. Bengal, Pt. 2, Nat. Hist. 66: 330. 1897

Type: Singapore, Bukit Mandai, 1892, H.N. Ridley 3835 (K barcode K000786153, lectotype designated here).

4.10 Terminalia subspathulata King, J. Asiat. Soc. Bengal, Pt. 2, Nat. Hist. 66: 332. 1897

Type: Peninsular Malaysia, Perak, Kinta, Gopeng, July 1883, Dr King's Collector 4529 (K barcode K000786156, lectotype designated here; isolectotypes BM, SING $(\times 2)$ barcodes SING0055425, SING0055427).

4.11 Terminalia triptera Stapf, Bull. Misc. Inform. Kew 1895: 103.1895

Type: Peninsular Malaysia, Langkawi, September 1890, C. Curtis 1684 (lectotype K barcode K000786162, lectotype designated here; isolectotype SING barcode SING0055430).

(=) Terminalia nigrovenulosa Pierre ex Gagnep., Not. Syst. 3: 285. 1914.

Type: Vietnam, ad pedes montis Mu Xoai, October 1866, L. Pierre 5011 (P barcode P02286589, lectotype selected by Lecompte (1969: 92).

(=) Terminalia nigrovenulosa Pierre ex Gagnep. var. gracilior Gagnep., Fl. Gen. Indo-Chine 2: 756. 1920
Type: Vietnam, Delta du Mè-Không, 1875-1877, J.H.A.J. Harmand s.n. (P barcode P04716917, lectotype designated here; isolectotypes $\mathrm{K}$ barcode K001129732, P barcodes P04716912, P04717014).

(=) Terminalia obliqua Craib, Bull. Misc. Inform. Kew 1912: 153. 1912.

Type: Thailand, Sriracha, Nawng Kaw, 19 September 1911, A.F.G. Kerr 2073 (K barcode K000786152, lectotype designated here; isolectotypes $\mathrm{BM}$ barcode BM000947100, E barcode E00284575, K barcocde K001129733, TCD barcode TCD0016766).

(=) Terminalia tripteroides Craib, Bull. Misc. Inform. Kew 1912: 152. 1912.

Type: Thailand, Meh Ping, Muang Hawt, 6 September 1911, A.F.G. Kerr 2010A (K barcode K000786151, lectotype designated here; isolectotypes $\mathrm{BM}$, E barcode E00284577, K no barcode, L barcode L0009816, TCD barcode TCD0016755).

(=) Terminalia hainanensis Exell, Sunyatsenia 2: 1. 1934. Type: China, Hainan, [Gnai Yuen,] 5 Jul 1933, F.C. How 70938 (BM barcode BM000947030, lectotype designated here; isolectotypes A barcodes A00068634, A00068635, B barcode B 10 0272120, IBK barcode IBK00190688, K barcode K000786127, NAS barcode NAS00047726, NY barcode NY00245994, P barcode P02286590, PE barcode PE00994592, US barcode US00117594).

Notes

An entry for Terminalia inguovenulosa Pierre is found in Lanessan (1886: 315), but as the only description presented is a vague size of the tree, the name is not validly published. Gagnepain (1916) indicated that Lanessan's name was a nomen nudum and that it was an error for Terminalia nigrovenulosa. Gagnepain effectively validated T. nigrovenulosa by providing a diagnosis comparing it with Terminalia oliveri Brandis. Terminalia triptera Stapf has priority over T. nigrovenulosa Pierre ex Gagnep. and is here accepted as the correct name for the species.

\section{ACKNOWLEDGEMENTS}

I am very grateful to Otakar Šida (PR), Uta Grünert (TUB) and Christian Bräuchler (W) for information on specimens. 


\section{REFERENCES}

Byng JW. 2014. The flowering plants handbook. Hertford: Plant Gateway Ltd.

Byrnes NB. 1977. A revision of Combretaceae in Australia. Contr Queensland Herb. 20:1-88.

Chen J, Turland NJ. 2007. Combretaceae. Fl China 13: 309-320.

Du Puy DJ, Telford IRH. 1993. Combretaceae. Fl Aust. 50: 243-247.

Don G. 1827. A review of the genus Combretum. Trans Linn Soc London. 15: 412-441.

Exell AW. 1931. The genera of Combretaceae. J Bot. 69: 113-128.

Exell AW. 1954. Combretaceae. Fl Males. 4:533-589.

Exell AW, Stace CA. 1966. Reorganisation of the genus Quisqualis. Bol Soc Brot, Sér 2. 38:139-143.

Exell AW, Stace CA. 1966. Revision of the Combretaceae. Bol Soc Brot, Sér 2. 40:1-25.

Fischer CEC. 1932. The Koenig collection in the Lund Herbarium. Bull Misc Inform, Kew. 1932: 49-76.

Gagnepain F. 1916. Quelques Combrétacées nouvelles. Not Syst. 3: 284-289.

Gangopadhyay M, Chakrabarty T. 1997. The family Combretaceae of Indian Subcontinent. J Econ Taxon Bot. 221:281-364.

Guillaumin A, Beauvisage G. 1914. Species montrouzieranae seu enumeratio plantarum in Nova Caledonia terrisque adjacentibus a R.P. Montrouzier lectarum. Publ Soc Linn Lyon. 38: 75-130.

Hepper FN. 1976. The West African herbaria of Isert and Thonning. Kew: Bentham-Moxon Trust.

Jordaan M, van Wyk AE., Maurin O. 2011. Generic status of Quisqualis (Combretaceae), with notes on the taxonomy and distribution of Q. parviflora. Bothalia. 41: 161-169.

Kochummen KM. 1972. Combretaceae. Tree Fl Malaya. 1:172-178.

de Lanessan J.-L. 1886. Les plantes utiles des Colonies françaises. Paris: Imprimerie Nationale.

Lecompte O. 1969. Combretaceae R. Brown. Fl Cambodge Laos Vietnam. 10:3-119.

Maurin O, Chase MW, Jordaan M, van der Bank M. 2010. Phylogenetic relationships of Combretaceae inferred from nuclear and plastid DNA sequence data: implications for generic classification. Bot J Linn Soc. 162: 453-476.

Maurin O, Turner IM, Boatwright JS, Christenhusz MJM. 2020. New combinations in Combretaceae subtribe Combretinae from Africa and Asia. Phytotaxa. 451: 231-237.

Merrill ED. 1917. An interpretation of Rumphius's Herbarium amboinense. Manila: Burea of Printing.
Nanakorn W. 1985. The genus Terminalia (Combretaceae) in Thailand. Thai For Bull Bot. 15: 59-107.

Nanakorn W. 1986. The genus Combretum (Combretaceae) in Thailand. Thai For Bull Bot. 16: 154-204.

Persoon HC. 1805. Synopsis plantarum. Volume 1.

Ridley HN. 1922. Flora of the Malay Peninsula. Volume 1.

Roxburgh W. 1814. Hortus bengalensis. Serampore: Mission Press.

Roxburgh 1832. Flora indica. Calcutta: W. Thacker \& Co.

Stace CA. 2007. Combretaceae. Fam Gen Vasc Pl. 9: 67-82

Stace CA. 2010. Combretaceae. Fl Neotrop Monogr. 107:1-369.

Tulasne R-B. 1856. Florae madagascariensis fragmenta. Ann Sci Nat. Sér. 4 6: 75-138.

Turland NJ, Wiersema JH, Barrie FR, Greuter W, Hawksworth DL, Herendeen PS, Knapp S, Kusber WH, Li DZ, Marhold K, May TW, McNeill J, Monro AM, Prado J, Price MJ, Smith GF. 2018. International Code of Nomenclature for algae, fungi, and plants (Shenzhen Code) adopted by the Nineteenth International Botanical Congress Shenzhen, China, July 2017. Regnum Vegetabile 159. Glashütten: Koeltz Botanical Books.

Turner IM. 2014. John Fleming, William Roxburgh and the Catalogue of Indian Medicinal Plants and Drugs. Kew Bull. 69: 9489.

Wickens GE. 1973. Flora of Tropical East Africa. Combretaceae, 1-99.

Wickens GE. 1990. 91. Combrétacées. Fl. Mascareignes 91:1-13. 